\title{
Lost in Translation?
}

$\mathrm{T}$ he definition of translational research refers to the steps between fundamental discovery and its application in clinical medicine with the goal of improving patient care and realizing the potential of biomedical discoveries for their benefit. For example, the cloning of a specific molecule such as the G-protein-calcium-sensing receptor followed by the approval just $10 \mathrm{y}$ later of a drug that acts specifically on this G-protein-calcium-sensing receptor (cinacalcet) which is now widely used for the treatment of secondary hyperparathyroidism in patients treated with dialysis (1).

Unfortunately, despite significant advances in the molecular understanding of many diseases, there are still big gaps in the application of these developments to clinical practice and, in particular, to pediatric medicine. The IGF receptor has been recognized as central to cancer growth for over two decades, but targeted antibodies are just being tested against it in malignant diseases (2). The molecular basis for sickle cell anemia was described four decades ago but has yet to be translated to a modern therapy (3). Indeed, basic scientists provide clinicians with new tools that may potentially be applicable in patients and clinical investigators continue to make novel observations about the specific responses of patients to therapies related to pharmacogenomics, ethnic and social background, and age, thereby stimulating new questions for basic investigators (4).

However, there are growing barriers between clinical and basic science investigators together with the increasing complexities involved in the conduct of clinical research that are making more difficult to translate new knowledge into clinical practice and back again to the bench. These issues are even more relevant in pediatrics because childhood diseases are less common and all drugs are initially approved by the FDA in adults, and later reviewed for children. However, the most important barrier to effective translation of basic discoveries relevant to children is that there is a wide range of variability between institutional review boards among major academic centers that negatively impact pediatric research. The current training of the next generation of clinical translational investigators is fragmented and not fully connected to the rapid development of genomic technologies. Thus, there is a need for training investigators on a wide range of skill sets that range from biomedical to behavioral sciences and such training will require a dedicated and structured curriculum.

Because of the growing barriers between clinical and basic research, together with the increasing complexity of conducting clinical research, the National Institutes of Health has initiated a process of reengineering the development of a new discipline of clinical and translational science under the umbrella of the Clinical and Translational Science Awards (CTSA). The purpose of the CTSA program is to assist institutions to create a new home for Clinical and Translational Science by integrating basic, translational, and clinical investigators with community clinicians to facilitate the development of new interactions, programs, and research projects. When fully implemented by 2012, approximately 60 institutions will be linked together to energize the discipline of clinical and translational science. The CTSA will create the appropriate environment for education and training the next generation of clinical and translation investigators. These new trainees will have the appropriate expertise that will allow them to build effective interdisciplinary science teams. The National Institutes of Health created a body to provide oversight to the pediatric aspects of the CTSA effort (5). The question remains, however, whether this effort will allow the translational effort to catch up with the momentum enjoyed by basic research (6). The amount of money dedicated by the National Institutes of Health to clinical/translational research remained dwarved by the funds dedicated to basic research and discovery. Thus, continued efforts to galvanize the communities of researchers and clinicians, particularly in the pediatric realm are still needed (7) and we must support initiatives at the National Institutes of Health and congress as well as at the academic grass root level, which will insure that children end up benefiting from the tremendous advances of 21 st century science. - Isidro B. Salusky and Pinchas Cohen

\section{REFERENCES}

1. Stewart AF 2004 Translational implications of the parathyroid calcium receptor. N Engl J Med 351:324-326

2. Osborne R 2008 Commercial interest waxes for IGF-1 blockers. Nat Biotechnol 26:719-720

3. Platt OS 2008 Hydroxyurea for the treatment of sickle cell anemia. N Engl J Med 358:1362-1369

4. Wilkinson GR 2005 Drug metabolism and variability among patients in drug response. N Engl J Med 352:2211-2221

5. Kon AA 2008 Real pragmatism, kids, and the Clinical and Translational Science Award (CTSA). Am J Bioeth 8:45-47

6. Butler D 2008 Translational research: crossing the valley of death. Nature 453:840-842

7. Zerhouni EA 2005 Translational and clinical science-time for a new vision. N Engl J Med 353:1621-1623 